\title{
Exactly solvable model of a highly efficient thermoelectric engine
}

\author{
Martin Horvat ${ }^{*}$ and Tomaž Prosen ${ }^{\dagger}$ \\ Department of Physics, Faculty of Mathematics and Physics, University of Ljubljana, Jadranska 19, SI-1000 Ljubljana, Slovenia \\ Giulio Casati \\ Center for Nonlinear and Complex Systems, Via Vallegio 11, 22100 Como, Italy \\ (Received 6 April 2009; published 31 July 2009)
}

\begin{abstract}
We propose a simple classical dynamical model of a thermoelectric (or thermochemical) heat engine based on a pair of ideal gas containers connected by two unequal scattering channels. The model is solved analytically and it is shown that a suitable combination of parameters can be chosen such that the engine operates at Carnot's efficiency.
\end{abstract}

In the frame of nonequilibrium thermodynamics a heat engine is a machine generating work while exchanging heat with two heat baths at different temperatures $T_{1}$ and $T_{2}$. The usual goal in a construction of realistic heat engines is to increase the efficiency as far as possible toward the theoretical upper limit $\eta_{\text {carnot }}=1-T_{1} / T_{2}$, assuming $T_{2}>T_{1}$. We are here interested in an engine without moving mechanical parts, i.e., which could operate in a nonequilibrium steady state, such as a thermoelectric or thermochemical couple. Such an engine-or a refrigerator if the operation is reversed-would have immense practical advantages over piston or compressor based engines for obvious reasons, also due to possibilities of drastic miniaturization [1].

Here we present an abstract model of a heat engine that can mimic the essential features of a realistic heat engine based on the thermoelectric effect [2-4] and which can be treated and solved analytically. It is based purely on deterministic classical dynamics and stochastic baths. The model is composed of two thermochemical reservoirs of ideal gas of equal point particles connected by two one-dimensional wires indexed by $i \in\{1,2\}$. In the middle of each wire we place a deterministic and energy conserving scatterer, which either reflects or transmits the particle depending on its kinetic energy $\epsilon$. This behavior is completely described by the transmission function $\tau_{i}(\epsilon) \in\{0,1\}$ of the $i$ th scatterer. We use units in which particle mass $m$, particle charge $e$, and Boltzmann constant $k_{\mathrm{B}}$ equal $m=e=k_{\mathrm{B}}=1$. In this Rapid Communication we show that in the steady state a nonvanishing circular particle current exists only if the transmission functions are energy dependent. Then we show that for a suitable combination of parameters the engine operates in a reversible way with the Carnot's efficiency.

The scheme of the heat engine is shown in Fig. 1. In the wires we introduce bias forces $\vec{E}_{i}$ (say electric fields), which can be described by bias voltages $U_{i}$ or any other form of external potential energy which can be used to extract useful work. In the stationary state, at some temperature difference, there is a nonzero (circular) particle current in the wires that,

\footnotetext{
*martin.horvat@fmf.uni-lj.si

†tomaz.prosen@fmf.uni-lj.si

†iulio.casati@uninsubria.it
}

by climbing against the electric potential, can perform useful work. In the left (right) reservoir, the particles are at chemical potentials $\mu_{\mathrm{L}}\left(\mu_{\mathrm{R}}\right)$ and temperature $T_{\mathrm{L}}\left(T_{\mathrm{R}}\right)$ (here we assume $T_{\mathrm{L}}>T_{\mathrm{R}}$ ) and are effused into the wires with the injection rates $p_{i} \gamma_{\mathrm{L}}\left(p_{i} \gamma_{\mathrm{R}}\right)$ into the first $i=1$ and the second $i$ $=2$ channel, respectively, where $p_{i} \in[0,1], p_{1}+p_{2}=1$, represent the relatives openings into the two channels. We note that both in the reservoirs and in the channels the motion of particles is assumed to be (quasi) one dimensional, so we consider a single component of the velocity. The injection rates are connected to the chemical potentials $\mu_{\nu}$ and inverse temperatures $\beta_{\nu}=1 / T_{\nu}$ via the formula

$$
\mu_{\nu} \beta_{\nu}=\ln \left(C \beta_{\nu} \gamma_{\nu}\right), \quad \nu \in\{\mathrm{L}, \mathrm{R}\},
$$

where $C$ is a constant depending only on properties of particles and on geometry of the reservoir opening [4]. The velocity $v$ of effused particles is distributed in each side according to a canonical distribution

$$
P_{\nu}(v)=\beta_{\nu} v e^{-\beta_{\nu} v^{2} / 2} \theta\left(\sigma_{\nu} v\right),
$$

where $\theta(v)=(1: v \geq 0 ; 0$ : otherwise $)$ is the unit step function, and $\sigma_{\mathrm{L}}=1, \sigma_{\mathrm{R}}=-1$. In the steady state, the particle currents $j_{\rho, i}$ within $i$ th wire and the heat currents [2] exchanged with the $\nu$-side bath and $i$ th wire $\left.j_{q, i}\right|_{\nu}$ are given by

$$
j_{\rho, i}=p_{i}\left(\gamma_{\mathrm{L}} t_{\mathrm{L}, i}-\gamma_{\mathrm{R}} t_{\mathrm{R}, i}\right)
$$

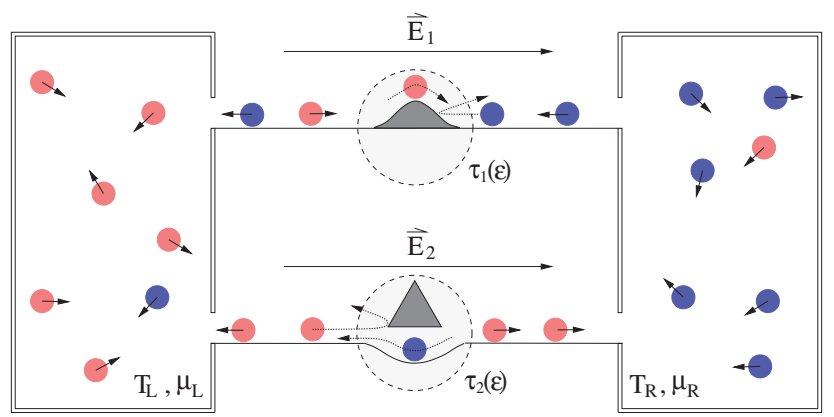

FIG. 1. (Color online) Schematic figure of the heat engine. The possible deterministic scattering mechanisms are depicted symbolically. Red (light gray) and blue (dark gray) circles schematically represent hot and cold particles, respectively. 

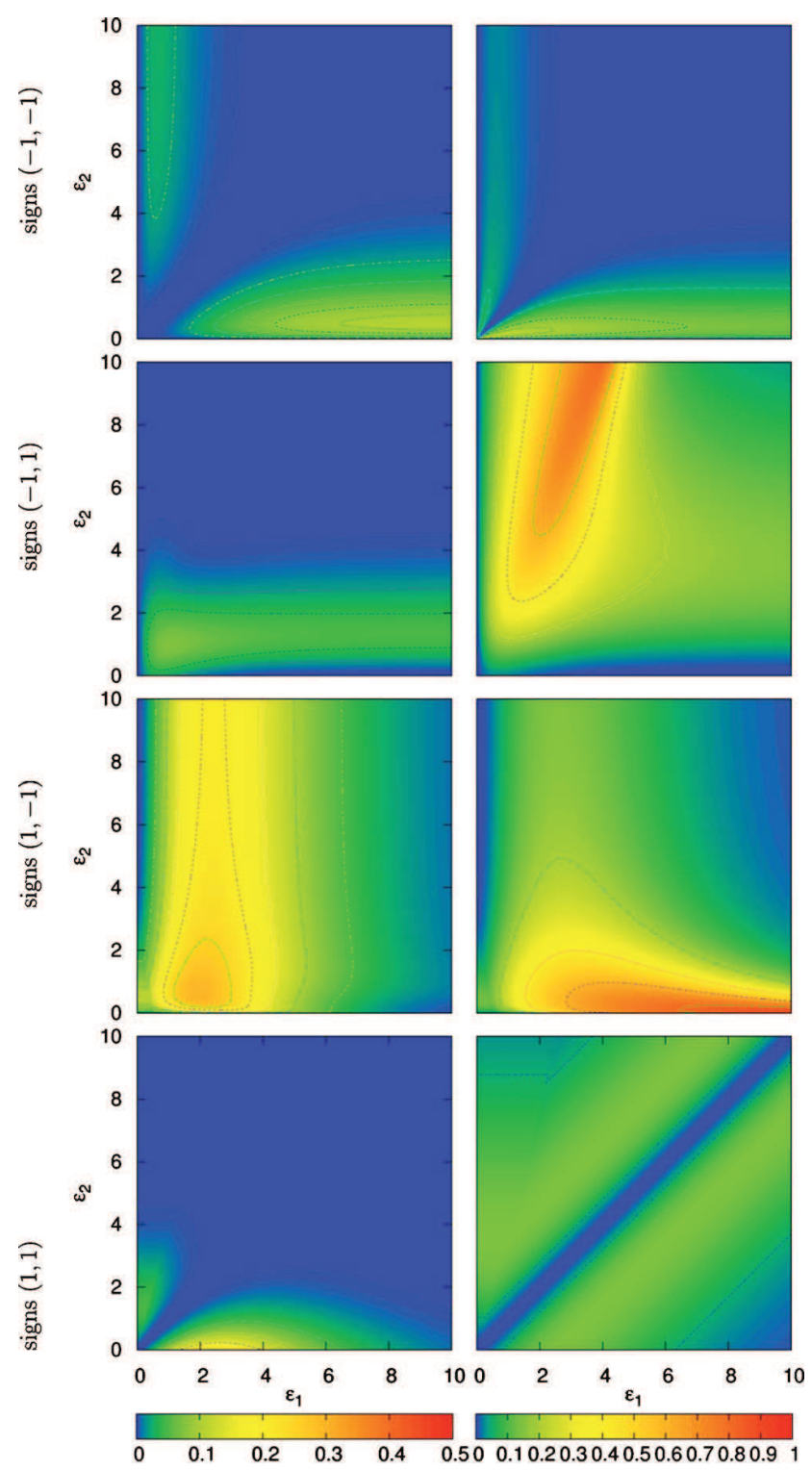

(a)

(b)

FIG. 2. (Color online) The relative power (a) $P^{*} / \eta_{\text {carnot }}^{2}$ and the relative efficiency (b) $\eta^{*} / \eta_{\text {carnot }}$ as functions of the energy thresholds $\epsilon_{i}$ for different sign configurations $\left(s_{1}, s_{2}\right)$ (indicated on the left) at unit mean injection rate $\bar{\gamma}=1$, equal channel openings $p_{i}$ $=1 / 2$, and bath temperatures $T_{\mathrm{L}}=2$ and $T_{\mathrm{R}}=0.5$.

$$
\begin{aligned}
& \left.j_{q, i}\right|_{\mathrm{L}}=p_{i}\left[\gamma_{\mathrm{L}} q_{\mathrm{L}, i}-\gamma_{\mathrm{R}}\left(q_{\mathrm{R}, i}+t_{\mathrm{R}, i} U_{i}\right)\right], \\
& \left.j_{q, i}\right|_{\mathrm{R}}=p_{i}\left[\gamma_{\mathrm{L}}\left(q_{\mathrm{L}, i}-t_{\mathrm{L}, i} U_{i}\right)-\gamma_{\mathrm{R}} q_{\mathrm{R}, i}\right] .
\end{aligned}
$$

Here we have introduced the transmission probability $t_{\nu, i}$ for a particle to transit from the $\nu$ side to the other side over the $i$ th wire, and its average kinetic energy $q_{\nu, i}$, explicitly defined in terms of the first two statistical moments of the energy distribution of the effused particles transmitted through the $i$ th wire,
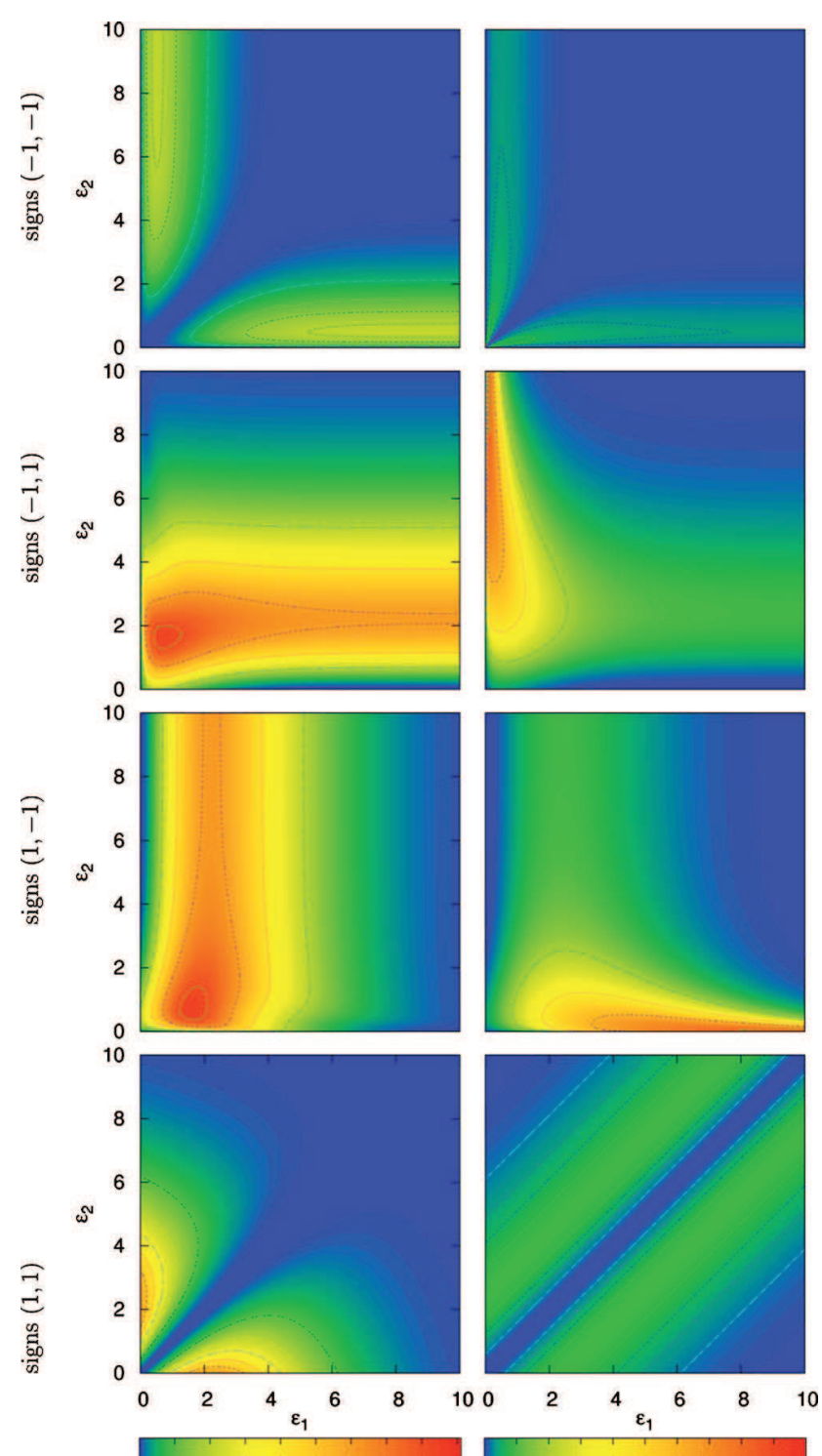

$\begin{array}{llllllllllllllll}0 & 0.1 & 0.2 & 0.3 & 0.4 & 0.5 & 0.6 & 0.7 & 0.8 & 0.9 & 0 & 0.10 .20 .30 .40 .50 .60 .70 .80 .9 & 1\end{array}$

(a)

(b)

FIG. 3. (Color online) The density plot of the relative power (a) $10 P^{*} / \eta_{\text {carnot }}^{2}$ and the relative efficiency (b) $\eta^{*} / \eta_{\text {carnot }}$ in the linear response regime, at the bath temperatures $T_{\mathrm{L}}=1.01$ and $T_{\mathrm{R}}=0.99$. (Other details the same as in Fig. 2.)

$$
\begin{aligned}
& \left(t_{\mathrm{L}, i}, q_{\mathrm{L}, i}\right)=\beta_{\mathrm{L}} \int_{\max \left\{0, U_{i}\right\}}^{\infty} d \epsilon e^{-\beta_{\mathrm{L}} \epsilon} \tau_{i}\left(\epsilon-\frac{U_{i}}{2}\right)(1, \epsilon), \\
& \left(t_{\mathrm{R}, i}, q_{\mathrm{R}, i}\right)=\beta_{\mathrm{R}} \int_{\max \left\{0,-U_{i}\right\}}^{\infty} d \epsilon e^{-\beta_{\mathrm{R}} \epsilon} \tau_{i}\left(\epsilon+\frac{U_{i}}{2}\right)(1, \epsilon) .
\end{aligned}
$$

The terms $U_{i} / 2$ in the arguments of transmission functions $\tau_{i}$ imply the assumption of linear potential and scatterers being in the middle of each wire. However, different assumptions (say of bias potential steps at the left/right of each scatterer) could be treated straightforwardly. By imposing the condition of stationarity $j_{\rho, 1}+j_{\rho, 2}=0$, we obtain, from Eq. (3), the injection rates $\gamma_{\mathrm{L}}$ and $\gamma_{\mathrm{R}}$, 


$$
1-\frac{\gamma_{\mathrm{L}}}{\bar{\gamma}}=\frac{\gamma_{\mathrm{R}}}{\bar{\gamma}}-1=\frac{p_{1}\left(t_{\mathrm{L}, 1}-t_{\mathrm{R}, 1}\right)+p_{2}\left(t_{\mathrm{L}, 2}-t_{\mathrm{R}, 2}\right)}{p_{1}\left(t_{\mathrm{L}, 1}+t_{\mathrm{R}, 1}\right)+p_{2}\left(t_{\mathrm{L}, 2}+t_{\mathrm{R}, 2}\right)},
$$

where $\bar{\gamma}=\left(\gamma_{\mathrm{L}}+\gamma_{\mathrm{R}}\right) / 2$ [5]. The resulting particle current in the wires,

$$
j_{\rho, 1}=2 \bar{\gamma} p_{1} p_{2} \frac{t_{\mathrm{L}, 1} t_{\mathrm{R}, 2}-t_{\mathrm{L}, 2} t_{\mathrm{R}, 1}}{p_{1}\left(t_{\mathrm{L}, 1}+t_{\mathrm{R}, 1}\right)+p_{2}\left(t_{\mathrm{L}, 2}+t_{\mathrm{R}, 2}\right)},
$$

determines the working power $P=j_{\rho, 1}\left(U_{1}-U_{2}\right)$ while the ingoing heat flux is equal to

$$
\begin{aligned}
Q & =\left.j_{q, 1}\right|_{\mathrm{L}}+\left.j_{q, 2}\right|_{\mathrm{L}} \\
& =-\frac{2 \bar{\gamma} \sum_{i, j=1}^{2} p_{i} p_{j} t_{\mathrm{L}, i} t_{\mathrm{R}, j}\left(U_{i}-S_{\mathrm{L}, i}+S_{\mathrm{R}, j}\right)}{p_{1}\left(t_{\mathrm{L}, 1}+t_{\mathrm{R}, 1}\right)+p_{2}\left(t_{\mathrm{L}, 2}+t_{\mathrm{R}, 2}\right)},
\end{aligned}
$$

where we have introduced the ratio $S_{\nu, i}=q_{\nu, i} / t_{\nu, i}$ (which is shown below to be connected to the Seebeck coefficient). The efficiency of the heat engine is then defined as $\eta$ $=P /|Q|$. Notice that the particle current $j_{\rho, 1}$, and so also the power $P$, is proportional to the determinant of the matrix of transmission coefficients $D=\operatorname{det}\left\{t_{\nu, i}\right\}=t_{\mathrm{L}, 1} t_{\mathrm{R}, 2}-t_{\mathrm{L}, 2} t_{\mathrm{R}, 1}$. The optimal performance of the heat engine for a given configuration of temperatures and scatterers is obtained by finding appropriate fields $U_{1}$ and $U_{2}$ which maximize $\eta\left(U_{1}, U_{2}\right)$. This can be done, in general, only numerically since it requires a solution of coupled transcendental equations.

Note the following important observation: $D=0$, and hence the currents vanish, despite nonvanishing temperature difference, if the scatterers are energy independent $\tau_{i}(\epsilon)$ $\equiv$ const. This fact is a simple consequence of time-reversal properties of individual deterministic trajectories which connect the two baths and remains valid for scattering channels in higher dimension (e.g., like in Ref. [4]).

Our ideas are demonstrated in a heat engine with bias voltage only in the first wire $\left(U_{2}=0\right)$ and for the simplest nontrivial, steplike, transmission functions

$$
\tau_{i}(\epsilon)=\theta\left(s_{i}\left(\epsilon-\epsilon_{i}\right)\right) .
$$

The direction of the steps, at the energy thresholds $\epsilon_{i}$, is determined by the signs $s_{i} \in\{1,-1\}$. Simple mechanical realizations for both signs $s_{i} \in\{1,-1\}$ are schematically depicted in Fig. 1. We numerically determine the potential $U_{1}$ that maximizes the efficiency for a given configuration of scatterers. The optimal efficiency $\eta^{*}=\max _{U_{1}} \eta$ and the corresponding power $P^{*}$ are shown in Fig. 2. In the case $s_{1}$ $=s_{2}=-1$ the scatterers transmit only slow enough particles. The regions of high power and high efficiency overlap and are positioned almost symmetrically near the axes. The exact symmetry is broken because the electric field is only applied to the first wire. The cases $s_{1}=-s_{2}=-1$ and $s_{1}=-s_{2}=1$ describe a similar situation, where one scatterer transmits the fast particles and the other scatterer transmits slower ones. This case is the most efficient and $\eta$ here may nearly approach $\eta_{\text {carnot }}$. However, as expected, the regions of high efficiency and high power only slightly overlap. In the last case $s_{1}=s_{2}=1$ the scatterers only transmit fast enough par- ticles. The region of high efficiency is located parallel to the line $\epsilon_{1}=\epsilon_{2}$. The highest power is obtained for energy steps matching the bath temperatures $\epsilon_{1}=T_{\mathrm{L}}$ and $\epsilon_{2}=T_{\mathrm{R}}$. A detailed analysis of the relaxation process shows that the convergence time to the nonequilibrium steady state is strictly finite for nonvanishing bias potentials and is given by $\tau^{*} \sim 1 / \min \left\{\left|U_{1}\right|,\left|U_{2}\right|\right\}$.

In the following we show that the results drastically simplify in the linear regime of small relative temperature difference. In this regime the meaningful bias potentials are also small and we may approximate the exact particle and heat fluxes in the wires with their linear expansions in the temperature difference $T_{\mathrm{L}}-T_{\mathrm{R}}$, injection rate difference $\delta \gamma=\gamma_{\mathrm{R}}$ $-\gamma_{\mathrm{L}}$, and potentials $U_{i}$. Expressing $\delta \gamma$ with the chemical potential difference $\delta \mu=\mu_{\mathrm{R}}-\mu_{\mathrm{L}}$, setting $\mu_{\mathrm{R}}+\mu_{\mathrm{L}}=0$, we write the particle and heat fluxes in the linear response limit as

$$
\begin{aligned}
& j_{\rho, i}=p_{i} \bar{\gamma}\left[-\beta g_{i}\left(U_{i}+\delta \mu\right)+h_{i} \delta \beta\right], \\
& j_{q, i}=p_{i} \bar{\gamma}\left[-\beta h_{i}\left(U_{i}+\delta \mu\right)+k_{i} \delta \beta\right],
\end{aligned}
$$

where $\beta=\left(\beta_{\mathrm{L}}+\beta_{\mathrm{R}}\right) / 2$ and $\delta \beta=\beta_{\mathrm{R}}-\beta_{\mathrm{L}}$. The expansion coefficients $g_{i}, h_{i}$, and $k_{i}$ are statistical moments of a canonical energy distribution of particles that are transmitted over the $i$ th wire,

$$
\left(g_{i}, h_{i}, k_{i}\right)=\beta \int_{0}^{\infty} d \epsilon e^{-\beta \epsilon} \tau_{i}(\epsilon)\left(1, \epsilon, \epsilon^{2}\right),
$$

and depend only on the transmission function and temperature. Notice that in the linear response limit the heat fluxes at the left and right sides are equal in contrast to the general (nonlinear) case [Eqs. (4) and (5)]. The coefficients $g_{i}$ and $h_{i}$ represent the average transmission probability of particles across the $i$ th wire and their average energy at zero bias fields. Instead of $h_{i}$ and $k_{i}$ it is more convenient to work with the average energy per particle $S_{i}=h_{i} / g_{i}$ and the coefficient $K_{i}=k_{i}-g_{i} S_{i}^{2}$. Note that $\beta p_{i} \bar{\gamma} g_{i}, \beta^{2} p_{i} \bar{\gamma} K_{i}$, and $\beta S_{i}$ can be interpreted as the particle conductance, the heat conductance, and the Seebeck coefficient, respectively. By imposing the stationarity condition $j_{\rho, 1}+j_{\rho, 2}=0$ we obtain the difference of the chemical potentials between baths:

$$
\delta \mu=-\frac{\xi\left(p_{1} g_{1} S_{1}+p_{2} g_{2} S_{2}\right)+p_{1} g_{1} U_{1}+p_{2} g_{2} U_{2}}{p_{1} g_{1}+p_{2} g_{2}},
$$

where $\xi=-\delta \beta / \beta$ is the relative temperature difference which is related to the Carnot efficiency $\eta_{\text {carnot }}=|\xi|$.

Let us now introduce the auxiliary quantities: difference of energies per particle in the two wires $\Delta S=S_{2}-S_{1}$, difference of potentials $\delta U=U_{2}-U_{1}$, transmission probability through both wires $G=\left[\left(p_{1} g_{1}\right)^{-1}+\left(p_{2} g_{2}\right)^{-1}\right]^{-1}$, and the figure of merit of the heat engine efficiency [6]

$$
y=\frac{G(\Delta S)^{2}}{p_{1} K_{1}+p_{2} K_{2}}>0 .
$$

We can now write the particle current in the first wire $j_{\rho, 1}$ and the ingoing heat flux $Q=j_{q, 1}+j_{q, 2}$ elegantly as 


$$
\begin{gathered}
j_{\rho, 1}=\beta \gamma G(-\delta U+\xi \Delta S), \\
Q=\beta \gamma G\left[\Delta S \delta U-\xi(\Delta S)^{2}(1+1 / y)\right],
\end{gathered}
$$

whereby the power and the efficiency of the heat engine are $P=j_{\rho, 1} \delta U$ and $\eta=P /|Q|$, respectively. Notice that all expressions just depend on the potential difference $\delta U$. In the linear response regime the potential that maximizes the efficiency can be found analytically by solving the equation $\partial \eta / \partial(\delta U)=0$. The explicit solutions are

$$
\begin{aligned}
\delta U^{*} & =\xi \Delta S[1-(\sqrt{1+y}-1) / y], \\
j_{\rho, 1}^{*} & =\xi \bar{\gamma} \beta G \Delta S(\sqrt{1+y}-1) / y, \\
Q^{*} & =-\xi \bar{\gamma} \beta G(\Delta S)^{2} \sqrt{1+y} / y,
\end{aligned}
$$

yielding the optimal efficiency (equivalent to Eq. (14) of [6])

$$
\eta^{*}=\frac{P^{*}}{\left|Q^{*}\right|}=\eta_{\text {carnot }}\left[1+\frac{2}{y}(1-\sqrt{1+y})\right],
$$

with the corresponding power $P^{*}=j_{\rho, 1}^{*} \delta U^{*}$. The relative optimal efficiency $\eta^{*} / \eta_{\text {carnot }}$, as expected, depends only on $y$ and is monotonic in the latter. Therefore it is meaningful to treat $y$ as the figure of merit of heat engine efficiency. We note that if the transmission functions are given by Eq. (11), results (19)-(22) are explicit as all the expressions are explicit rational functions of the moments of the Laplace transform of the transmission functions (14), which in turn are simple algebraic functions of $\beta, \epsilon_{i}$ and $\exp \left(-\beta \epsilon_{i}\right)$. From Eqs. (19) and (20) we can recognize that $P^{*} \propto \eta_{\text {carnot }}^{2}$, and consequently, in the linear response regime the power output is rather small. The optimal efficiency and the corresponding power as function of $\epsilon_{1}$ and $\epsilon_{2}$ in linear response regime are shown in Fig. 3. They are quite similar to those obtained in the nonlinear regime shown in Fig. 2. The important difference between nonlinear and linear regime results is that the latter only depends on the difference $\delta U$ of bias potentials and can be made temperature independent by expressing the energy steps in the transmission function $\tau_{i}(\epsilon)$ with the parameters $r_{i}=\beta \epsilon_{i}$. Consequently, the power $P^{*}$ and the efficiency $\eta^{*}$ as function of $\left(\epsilon_{1}, \epsilon_{2}\right)$ in the cases $s_{1}=-s_{2}=1$ and $s_{1}=-s_{2}=-1$ are exactly symmetric with respect to exchange of the parameters $\epsilon_{i}$. These cases are also the most efficient.
The region of high efficiency is squeezed toward the $\epsilon_{2}$ or $\epsilon_{1}$ axes in the cases $s_{1}=-s_{2}=1$ and $s_{1}=-s_{2}=-1$, respectively.

We have performed exact analytical calculations of expressions (19)-(22) for the case of equal channel openings $p_{i}=1 / 2$. In the cases $s_{1}=s_{2}=1$ and $s_{1}=s_{2}=-1$ the maximal efficiency $\eta_{\max }=\max _{r_{1}, r_{2}>0} \eta^{*}$ is reached at finite $\left(r_{1}, r_{2}\right)$ and is equal to $\eta_{\max } \stackrel{.}{=} 0.066 \eta_{\text {carnot }}$ and $\eta_{\max } \doteq 0.091 \eta_{\text {carnot }}$, respectively. However, in the case $s_{1}=-s_{2}=1$ (and similarly for $s_{1}=-s_{2}=-1$ ) we can reach the Carnot efficiency in the limit $r_{1} \rightarrow \infty$ following the curve $r_{2}^{2} \asymp 2 \exp \left(-r_{1}\right)$ along which the efficiency algebraically increases as $\eta^{*} \asymp \eta_{\text {carnot }}(1$ $\left.-2 r_{1}^{-1}\right)$, and the power exponentially decreases as $P^{*} \asymp 2 \eta_{\text {carnot }}^{2} \bar{\gamma} \beta^{-1} e^{-r_{1}}\left(r_{1}-1+\frac{5}{2 r_{1}}\right)$.

In conclusion, we have proposed a simple exactly solvable classical-mechanical model of thermoelectric (or better to say, thermochemical) heat engine. We presented closed form solutions for the steady state of the engine in linear and nonlinear regimes. A variable thermodynamic efficiency has been found, as a function of the system's parameters, which can become arbitrarily close to Carnot's in an appropriate regime.

Finally we would like to draw the reader's attention to the following point: it is possible to argue that our model is quite abstract in nature and therefore far from possible realistic implementations. We think on the contrary that this is the main advantage of our approach. After more than 50 years during which thermoelectric efficiency did not substantially increase we propose here to take a completely opposite point of view. Starting from fundamental microscopic equations and considering the most general schematized framework, we hope to understand the basic dynamical mechanisms which can lead to an increase in thermoelectric efficiency. In this spirit, the model discussed here is a step in this direction. In addition our model should be relevant for a theoretical description of nanoscopic heat engines, for example, a pair of thermoelectrically coupled quantum dots. However, our model would be a good approximation to the real system only in a rather restricted situation of (i) noninteracting charge carriers, (ii) negligible phonon contributions to heat transport, and (iii) coherence length longer than wires, which (iv) should be quasi-one-dimensional.

M.H. gratefully acknowledges the financial support by Slovenian Research Agency, grant Z1-0875-1554-08.
[1] G. Mahan, B. Sales, and J. Sharp, Phys. Today 50 (3), 42 (1997).

[2] S. R. de Groot and P. Mazur, Nonequilibrium Thermodynamics (North-Holland, Amsterdam, 1962).

[3] T. E. Humphrey and H. Linke, Physica E 29, 390 (2005).

[4] G. Casati, C. Mejia-Monasterio, and T. Prosen, Phys. Rev. Lett. 101, 016601 (2008).
[5] We note that the injection rates are simply related to the density of particles $\rho_{\nu}$ and temperatures $T_{\nu}$ in the baths, as $\gamma_{\nu}$ $\propto \rho_{\nu} \sqrt{T}$, with the proportionality factor depending only on the geometry of the channel (wire) openings [4]. However in order to make resulting expressions simpler we shall express them in terms of $\bar{\gamma}$.

[6] J. Wang, G. Casati, T. Prosen, and C.-H. Lai (unpublished). 